\title{
The case against primordial nucleosynthesis
}

\section{Geoffrey Burbidge}

\author{
Center for Astrophysics and Space Sciences, University of California, \\ San Diego, CA 92093-0424, USA \\ email: gburbidge@ucsd.edu
}

\begin{abstract}
It is shown that there is no underlying basic theory which will lead to the idea that the lightest isotopes were produced in an early universe. Everything depends on the choice of the initial ratio of the energy density of photons to baryons that is chosen (originally by Gamow and his colleagues). There is a clear alternative for the origin of the light isotopes. This is that they were generated by hydrogen burning in stars which produces the helium directly while ${ }^{2} D$ and ${ }^{7} \mathrm{Li}$ are produced by flare activity in stellar atmospheres. All of this activity takes place in the centers of active galaxies where matter is being created in a cyclic (quasi-steady) state universe.
\end{abstract}

Keywords. Early universe, cosmological parameters, nuclear reactions, nucleosynthesis, abundances

\section{Introduction}

It is nearly 60 years since Hoyle (1946) first proposed that the chemical elements are largely built by stellar nucleosynthesis. By 1957 more detailed work (Burbidge et al. (1957); Cameron (1957)) had shown that a strong case could be made for this hypothesis, and for the last 50 years the theory has been completely accepted and is one of the major pillars of astrophysics. The original proposal that all of the elements were made in the early universe, made by many leading physicists in the 1940ś and 1950ś was given up when it was realized that it was impossible to overcome the problems at mass 5 and 8 , and also because it was realized by a few astrophysicists that the observational evidence already showed that there were significant abundance differences between stars.

However, it was realized by the early pioneers, particularly Gamow and his colleagues, that with a suitable choice of parameters $\mathrm{D},{ }^{3} \mathrm{He},{ }^{4} \mathrm{He}$, and ${ }^{7} \mathrm{Li}$ could be built in an early universe, and since then much work has gone into getting agreement between theory and observation for these isotopes.

Nowadays, this work is often introduced as follows (I quote from a paper from David Tytler's group [Kirkman et al. (2000)]),

Big Bang nucleosynthesis is a cornerstone of modern cosmology. The standard theory $(S B B N)$ predicts the abundances of the light nuclei $\mathrm{H}, \mathrm{D},{ }^{3} \mathrm{He},{ }^{4} \mathrm{He}$, and ${ }^{7} \mathrm{Li}$ as a function of a single parameter, the cosmological baryon to photon ration $\eta=$ $\eta_{b} / \eta_{\gamma}$. The ratio of any two primodial abundances should yield a direct measurement of $\eta$ and the measurement of a third provides a test of the theory.

This is what a large majority of cosmologists now believe and this argument is used to support and propagate the view that the big bang cosmology is correct. But of course the statement I have quoted is not correct, primarily because there is no underlying theory.

In order to understand this, we have to go back to the early work of Gamow. It was already known at that time that the bulk of the known helium-some $25-30 \%$ by mass, could not have been made in stars seen in the galaxies. In those days it was thought that 
the Hubble constant was about ten times as large as it is known to be today and thus the age $\simeq H_{0}^{-1}$ was only about $2 \times 10^{9}$ years, and very little helium could have been made in that time.

Thus it was concluded that the helium must have originated in primordial nucleosynthesis. This required that the energy density of radiation in the early universe had to be very large. Until then, the reverse had always been assumed in Friedmann models. In such models $\mathrm{S}(\mathrm{t})$ (the scale factor) is proportional to $t^{1 / 2}$ so that $T_{9}=$ const. $^{-1 / 2}$. So long as the energy in the early universe is dominated by radiation this relation will hold. The next step was completely ad hoc. The mass density of stable non-relativistic particles $\rho_{b}$, explicitly neutrons and protons in the theory of 1950 , decreases with the expansion as $S^{-3}$ or $t^{-3 / 2}$. Alpher and Herman put the density $\rho_{b}=1.70 \times 10^{-2} t^{-3 / 2} \mathrm{gm}^{-3}$ the constant in this equation being the ad hoc step. But there is nothing in the theory which fixes the value of this numerical coefficient. It is chosen to make things come out right, -to make the calculated value of $\mathrm{Y}$ agree with the observed value. This is why the big bang theory cannot be claimed to explain the microwave background or to explain a cosmic helium value close to 0.25 . It is only an axiom of modern big bang cosmology, and the supposed explanation of the microwave background is a restatement of that axiom. Thus, in no sense, did the big bang theory predict the microwave background. $\dagger$ This would be true only if putting the factor $1.7 \times \overline{10^{-2}}$ is called a prediction. If we eliminate $t$ between the relations given above we find that

$$
\rho_{b}=1.51 \times 10^{-32} T^{3}
$$

which can be rounded off to $\rho_{b} \approx 1.51 \times 10^{-32} \mathrm{~T}^{3} \mathrm{gm}_{\mathrm{cm}}^{-3}$. Alpher and Herman put the mass density of the universe $\rho_{b} \approx 10^{-30} \mathrm{gm} \mathrm{cm}^{-3}$ and thus concluded that $\mathrm{T}$ must be about $5^{\circ} K$. Ten years later, when the Hubble constant had been further reduced, it appeared that $\rho_{b} \approx 10^{-29} \mathrm{gm}_{\mathrm{cm}}^{-3}$, and then both Gamow and Dicke suggested that $T \approx \times 15^{\circ} \mathrm{K}$. Of course these were gross overestimates.

What none of the physicists throughout this period were aware of, was that in 1941 McKellar (1941) had determined the temperature of the interstellar radiation from the spectra of the interstellar lines due to the molecules $\mathrm{CH}$ and $\mathrm{CH}^{+}$which Adams and later McKellar had detected in the spectra of stars. McKellar showed that if the radiation has black body form, $1.8^{\circ} \mathrm{K}<T<3.4^{\circ} \mathrm{K}$, which is in remarkable agreement with what was found later. The exact quote from McKellar is as follows:

Adams has kindly communicated to the writer his estimate of the relative intensity, in the spectrum of $\xi$ Ophiuchi, of the $\lambda R(0)$ interstellar line of the $\lambda 3883$ $C N$ band and the $\lambda 3874 R(1)$ line, as 5 to $1 . B_{-0} J^{\prime \prime}\left(J^{\prime \prime}+1\right)+\ldots$ has the value 0 and $3.78 \mathrm{~cm}^{-1}$ for the 0 and 1 rotational states and for the two lines (0) and $R(1)$ the values of the intensity factor $i$ are, respectively 2 and 4. Thus from (3) we find, for the region of space where the CN absorption takes place, the "rotational" temperature,

$$
T=2.3^{\circ} K
$$

If the estimate of the intensity of $R(0) / R(1)$ were off by 100 per cent, this value of the "rotational" temperature would not be changed greatly, $R(0) / R(1)=2.5$ giving $T=3.4^{\circ} \mathrm{K}$ and $R(0) / R(1)=10$ giving $T=1.8^{\circ} \mathrm{K}$.

$\dagger$ For $Y=0.24$ which is closer to the preferred current value of the fractional helium abundance, the constant $\eta$ is now close to $4.5 \times 10^{-2}$ 
When in 1965, Penzias and Wilson reported that they had directly detected the radiation (Penzias \& Wilson (1965)), and later Mather et al. $(1990,1994)$ showed that the radiation is of almost perfect black body form with $T=2.726^{\circ} \mathrm{K}$, they were richly rewarded. What I want to stress here is that while the black body nature of the radiation was predicted by the big bang theory, the numerical value of the temperature was not, and cannot be (see Turner (1993)) and since McKellar had already measured it, admittedly indirectly, it is a moot point as to whether or not the 1965 event truly was a major discovery. (If someone has already discovered a new phenomenon and published it, but the people most interested are unaware of the earlier discovery, how should credit be apportioned?) In truth no prediction was involved. But the psychological effect based on mistaken ideas concerning the prediction and discovery is one of the major reasons why the big bang is believed by so many.

Going back now to the primordial abundances: what is now being done is to put the observed temperature into the equation and derive a value for $\rho_{b}$. This is then compared with the value obtained from the nucleosynthesis calculations and observations involving $D, H e^{3}$ and $H^{4}$. Very good agreement can be reached between theory and observation for $\rho \approx 3 \times 10^{-31} \mathrm{gm}_{\mathrm{cm}}^{-3}$; so this is now called the observed baryonic mass fraction in the universe. This is a clear plus for the big bang cosmology. However, since the closure density in the big bang model $3 H_{0}^{2} / 8 \pi G$ is about $6.8 \times 10^{-30} \mathrm{gm}^{-3}$ (for $H_{0}=60 \mathrm{~km} \mathrm{sec}^{-1} \mathrm{Mpc}^{-1}$ ) this is only about $5 \%$ of the closure density.

While this discrepancy has been know for $\sim 30$ years, it is only in the last few years that this "missing" mass energy has been claimed first to be cold dark matter (CDM) and more recently cold dark matter and dark energy $(\Lambda C D M)$.

An elaborate "theory" (more appropriately a "scenario") of galaxy formation then rests on this belief that this missing mass is real, because only if CDM exists in a large measure is it possible to simulate galaxy formation at all. This is a classical example of "The Emperor has no clothes" syndrome. While a great deal of energy and money is being devoted by particle physicists to searches for the WIMPS which could conceivably be the basis for the dark matter, nothing has been found so far (cf. Science, 304, 950, 2004).

But, of course, none of this is necessary if we go back to the original observation of the $\mathrm{He} / \mathrm{H}$ ratio and take the position that the observed ratio is the result of hydrogen burning in stars. Then, of course, the whole of the mass must be baryonic. This leads us to one final point. If hydrogen burning was responsible for this ratio, an estimate can be made directly from observation of the energy released in this process. The mass density in the universe can be made directly from observation of the energy released in this process. The mass density in the universe can be determined from the masses of galaxies derived from their rotation curves and/or the velocity dispersion of the stars in galaxies, or of the galaxies in clusters. Both for individual galaxies and clusters it is assumed that the virial condition holds, so that in making this estimate we are assuming that some of the mass is dark. Putting in observed values for the space density of galaxies and a range of values of $\mathrm{M} / \mathrm{L}$, and a Hubble constant of $60 \mathrm{~km} \mathrm{sec}^{-1} \mathrm{Mpc}^{-1}$ and supposing that the ${ }^{4} \mathrm{He} / \mathrm{H}$ ratio is 0.24 , we obtain an energy density of the radiation $4.5 \times 10^{-13} \mathrm{erg} \mathrm{cm}{ }^{-3}$.

This energy will initially be released in hard photons (UV radiation) but ultimately, according to thermodynamic arguments, it will be degraded to black body radiation with $T \approx 2.75^{\circ}$. This is remarkably close to the measured value of $2.726^{\circ} \mathrm{K}$. This is either a pure coincidence, as it must be for those who believe in the big bang, or else it tells us that hydrogen burning was originally responsible for the CMB. In the cyclic universe model (Hoyle, Burbidge \& Narlikar (2000), Narlikar, Burbidge \& Vishwakarma (2005)) it is argued that it is due to hydrogen burning in the newly created galaxies and that 
intergalactic dust is responsible for the degradation to themal energy. Thus the CMB at the level detected is relic starlight from previous oscillations of the cyclic universe which has been thermalized. In this case there is no need to invoke primordial nucleosynthesis.

While this numerical agreement was mentioned in one or two earlier papers (cf. Fowler, Wagoner \& Hoyle (1967)) it was not described in detail until 1998 when Hoyle and I managed to get it published in the Astrophysical Journal (Burbidge \& Hoyle (1998)). The paper was earlier rejected by Phys. Rev. Letters whose referees were strong proponents of the big bang. In our paper we showed that it was possible to explain the origin of all of the isotopes including D and ${ }^{3} \mathrm{He}$ in stars. D is probably built up in stellar flares on the surfaces of stars and partly destroyed by mixing in stellar interiors. An observational fact following from this hypothesis is that it predicts the $\mathrm{D} / \mathrm{H}$ will be variable from one place in the galaxy to another, from galaxy to galaxy, and from QSO to QSO. But there really is no need to invoke a big bang. In other words there is no case for primordial nucleosynthesis based on which we observe. If you want to believe in a beginning then you can get the correct abundances by choosing the right parameters. But belief is not a theory.

Since none of the observations just described require an early universe, what are the alternatives ? Since the universe is expanding we can consider as possiblities a classical steady-state universe in which it was predicted that the universe is accelerating, but which has other difficulties, or a cyclic universe with a cycle period $\simeq 20$ Gyr (Hoyle, Burbidge \& Narlikar (2000), Narlikar, Burbidge \& Vishwakarma (2005)). Here we omit discussion of Milne's kinematic cosmology, though it should not be forgotten that Milne raised the problem of the particle horizon, in the classical big bang picture, and this is only claimed to be resolved now by recourse to an inflationary period.

I believe that the cyclic universe model which has just been referenced can give a good explanation of all the observations, and not a chosen few of them, as is the case with the majority who concentrate above all on fine details of the microwave background but are quite happy to believe in inflation (a nice idea without basic theory), non-baryonic matter (for which there is no observational evidence at all, but which is required to make galaxies in big bang cosmology) and what is called dark energy. Both big bang cosmology and the cyclic universe cosmology must deal with the problem of creation, (alternatively dark energy).

I consider it plausible that the creation process, which gives rise to new matter and energy, and stellar nucleosynthesis, is taking place in the nuclei of active galaxies.

\section{Summary}

If you want to believe in primordial nucleosynthesis, i.e. if you really believe in a big bang, you can choose parameters which will make observation and theory agree, but the argument is not based on basic theory.

But there is a perfectly legitimate alternative. There was no beginning, and the light elements are made originally following hydrogen burning in stars as new matter was being created in galactic nuclei in a cyclic universe. What this has going for it are the energetic arguments based simply on what we currently observe, and observations of active galaxies.

\section{References}

Burbidge, E.M., Burbidge, G., Fowler, W.A. \& Hoyle, F 1957, Rev. Mod. Phys. 29, 547

Burbidge, G. \& Hoyle, F. 1998, ApJ 509, L1 
Cameron, A.G.W. 1957, Reports Chalk River (Canada)

Fowler, W.A., Wagoner, R. \& Hoyle, F. 1967, ApJ 148, 3

Hoyle, F. 1946, MNRAS 106, 343

Hoyle, F., Burbidge, G. \& Narlikar, J.V. 2000, A Different Approach to Cosmology (Cambridge University Press)

Kirkman, D., Tytler, D., Burles, S., Lubin, D. \& O'Meara, J. 2000, ApJ 529, 655

Mather, J.C., Cheng, E.S., Eplee, R.E., et al. 1990, ApJ 354, L37

Mather, J.C., Cheng, E.S., Cottingham, D.A., et al. 1994, ApJ 420, 439

McKellar, A. 1941, Pub. Dominion Astrophysical Observatory 7, 25

Narlikar, J.V., Burbidge, G. \& Vishwakarma, R.G. 2005, submitted to Proc. Roy. Soc.A.

Penzias, A. \& Wilson, R.W. 1965, ApJ 142, 419

Turner, M. 1993, Science 262, 861 


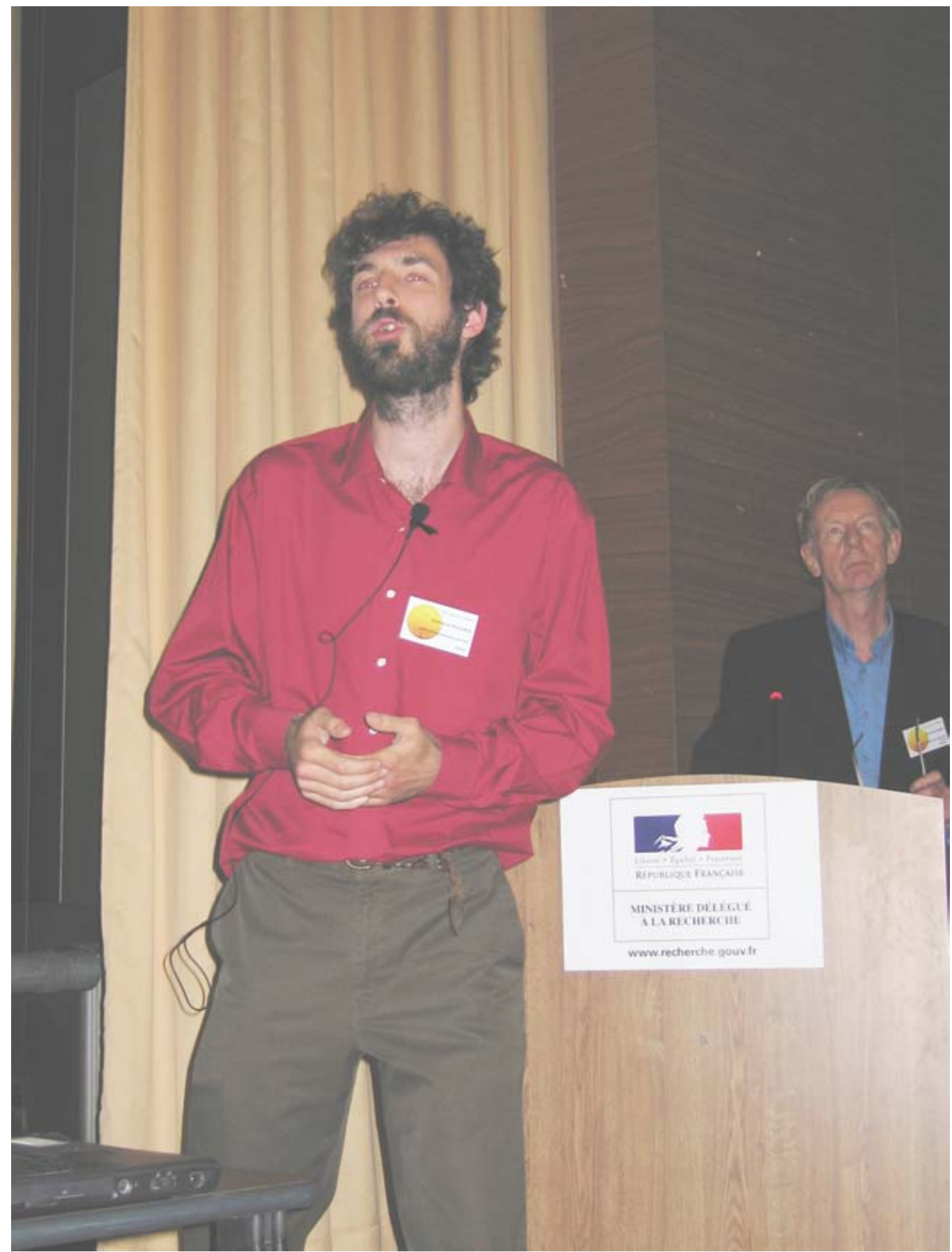

Emmanuel Rollinde, talking about the primordial lithium production, chaired by Poul E. Nissen. 